La Plata-Th 96/14

\title{
On the Path Integral Representation for Spin Systems
}

\author{
Daniel C. Cabra ${ }^{a}$, Ariel Dobry ${ }^{b}$, Andrés Greco $^{b}$ and \\ Gerardo L. Rossini ${ }^{a}$ \\ ${ }^{a}$ Departamento de Física, \\ Universidad Nacional de La Plata, \\ C.C. 67 (1900) La Plata, Argentina. \\ ${ }^{b}$ Departamento de Física, Facultad de Ciencias Exactas, Ingeniería y \\ Agrimensura and IFIR (CONICET-UNR). Av. Pellegrini 250, 2000 \\ Rosario, Argentina.
}




\begin{abstract}
We propose a classical constrained Hamiltonian theory for the spin. After the Dirac treatment we show that due to the existence of second class constraints the Dirac brackets of the proposed theory represent the commutation relations for the spin. We show that the corresponding partition function, obtained via the Fadeev-Senjanovic procedure, coincides with the one obtained using coherent states. We also evaluate this partition function for the case of a single spin in a magnetic field.
\end{abstract}




\section{Introduction}

The Heisenberg Model has been used for many years as a canonical model of magnetism in solids. After the discovery of High Tc superconductivity a renewed interest in this model emerged. This is principally due to the connection between the antiferromagnetic Heisenberg model and other models (Hubbard and $t-J$ models) [1] which are important to describe the electronic structure of the High Tc superconductors.

Although the Heisenberg model is quadratic in the spin operators it is highly non-trivial. What turns difficult the solution of the model are the complicated commutation relations among the spin components. This kind of commutation relations are indeed characteristic of systems described by Hubbard operators [2]. In particular the spin algebra $(s u(2))$ is the bosonic subalgebra of that spanned by the Hubbard operators in the so-called atomic representation of the $t-J$ model, $\operatorname{Osp}(2,2)$ [3].

When one is faced to a system with this commutation rules one usually implements some kind of decoupling using slave particles [四]. This new representation is formally exact and solves the above mentioned difficulty because the basic variables are conventional bosons and fermions. In particular a path-integral treatment is standard. However, in general, no exact calculation can be carried out thoroughly and some kind of approximation is needed (V.gr. mean field, large $s$, large $N$, etc.). A natural question which emerges in this framework (first decoupling and then approximating) is what is an artifact of the decoupling and what is not (see for example [5] for a recent discussion).

For the analysis of this question, a treatment of spin systems without the introduction of these fictitious slave particles would be of some importance. In particular, a path integral representation is a suitable formulation for this analysis. Indeed, such a path integral representation can be written using the coherent state method (also known as geometric quantization) [3, 6].

Although path integral methods have shown to be very powerful in various areas, the coherent state method is not a familiar subject in solid state physics. Moreover, as pointed in the literature «, 6, 7], there are some mathematical subtleties in the derivation of this path integral representation that prevent for a complete confidence on the results.

In this paper we present a constrained Hamiltonian system which after the Dirac treatment [8] reproduce the physics of the spin. Then we write 
down its Feynman path integral following the Fadeev-Senjanovic method for constrained Hamiltonian systems [9]. Our derivation is formally exact, independent of the coherent state method and valid irrespectively of the value of the spin s. Moreover, it has the virtue of setting the study of spin systems on canonical grounds. This is an important point in view of possible further applications of the formalism for spin $1 / 2$ systems.

Finally we explicitly calculate the free energy of a particle with fixed spin in an external magnetic field. The result gives an independent check for the validity of the expression of the partition function for all values of the spin. Besides, we think that the methods we use are very instructive and could be useful to develope approximation schemes for the Heisenberg model.

The paper is organized as follows: in section II we present the constrained Hamiltonian system and we perform the Dirac treatment. In section III we find the path integral representation for the quantum mechanics and the partition function. We calculate the partition function of one spin in Section IV. Finally a brief summary of our main results is given in section V.

\section{The constrained Hamiltonian system}

The starting point for our analysis is the following Hamiltonian

$$
H=H(\vec{n})
$$

and the additional set of primary constraints

$$
\begin{aligned}
& \Gamma_{1} \equiv P_{1}-s A_{1}(\vec{n}) \approx 0, \\
& \Gamma_{2} \equiv P_{2}-s A_{2}(\vec{n}) \approx 0, \\
& \Gamma_{3} \equiv P_{3}-s A_{3}(\vec{n}) \approx 0,
\end{aligned}
$$

where $\vec{P}$ is the canonical momentum associated to the vector $\vec{n}$ and

$$
\Gamma_{4} \equiv|\vec{n}|^{2}-1 \approx 0
$$


Equations (1-5) define a classical constrained Hamiltonian system in the Dirac sense [8]. Note that the constraints (2-4) are imposed in the weak sense.

The classical Hamiltonian is a general function of a vector $\vec{n}$. To simplify the notation we do not include lattice indices in the vector $\vec{n}$ but the following analysis is valid in the case where the spins live over a lattice. The Hamiltonian (1) is independent of the momentum $\vec{P}$ associated to the vector field $\vec{n}$. The corresponding momentum appears through the constraints (2-4). In these constraints we also have a general vector function $\vec{A}(\vec{n})$. In $(2-4) s$ is an arbitrary number. It will become the size of the spin after quantization. Finally, the constraint (5) indicate the normalization of the vector $\vec{n}$.

According to the Dirac procedure we have to define the following total Hamiltonian:

$$
H_{T}=H(\vec{n})+\sum_{i} \Gamma_{i} \lambda_{i}
$$

where the Lagrangian multipliers $\lambda_{i}$ are general functions of the coordinates and momenta.

The consistency conditions of the theory require the preservation in time of the constraints $(2-5)$

$$
\dot{\Gamma}_{i}=\left\{\Gamma_{i}, H_{T}\right\}_{P B} \approx 0
$$

In $(7)\{,\}_{P B}$ means the conventional Poisson Brackets. It is straightforward to show that the system (7) determines uniquely the Lagrange multipliers (the explicit form of these multipliers is not necessary for the rest of the paper). Therefore the constraints are preserved in time and secondary constraints are not generated. Moreover our theory is completely determined by the first class Hamiltonian (6) and the second class constraints (2-5) (there is no hidden gauge invariance in our system).

Due to the second class character of the constraints, the next step is the calculation of the Dirac brackets (DB). For any two classical quantities $A$, $B$, the Dirac bracket is defined by

$$
\{A, B\}_{D B}=\{A, B\}_{P B}-\left\{A, \Gamma_{i}\right\}_{P B} \Delta_{i j}\left\{\Gamma_{j}, B\right\}_{P B}
$$


where

$$
\Delta_{i j}\left\{\Gamma_{j}, \Gamma_{k}\right\}_{P B}=\delta_{i k}
$$

Using the $\mathrm{PB}$, it can be shown that the antisymmetric matrix $\Delta_{i j}^{-1}$ has elements

$$
\begin{aligned}
\Delta_{12}^{-1} & =s\left[\frac{\partial A_{2}}{\partial n_{1}}-\frac{\partial A_{1}}{\partial n_{2}}\right] \\
\Delta_{13}^{-1} & =s\left[\frac{\partial A_{3}}{\partial n_{1}}-\frac{\partial A_{1}}{\partial n_{3}}\right] \\
\Delta_{14}^{-1} & =-2 n_{1} \\
\Delta_{23}^{-1} & =s\left[\frac{\partial A_{3}}{\partial n_{2}}-\frac{\partial A_{2}}{\partial n_{3}}\right] \\
\Delta_{24}^{-1} & =-2 n_{2} \\
\Delta_{34}^{-1} & =-2 n_{3}
\end{aligned}
$$

In summary, we have a theory which is defined by a first class Hamiltonian (6) without arbitrary coefficients and the set of second class constraints (2)(5).

At this point the constraints are imposed as strong equations between coordinates and momenta. Also the PB are replaced by the DB.

\section{Quantization and Statistical Mechanics}

The canonical quantization can be now performed in the standard way. The Hamiltonian (6) have to be considered as the Hamiltonian operator. The constraints (2-5) become strong equations between operators and the commutation relations are obtained from the DB. The commutation relations between two operators $\hat{O}_{1}$ and $\hat{O}_{2}$ are given by:

$$
\left[\hat{O}_{1}, \hat{O}_{2}\right]=\imath \hbar\left\{O_{1}, O_{2}\right\}_{D B}
$$

The vector field $\vec{A}(\vec{n})$ is not specified yet. If we choose it as: 


$$
\nabla_{\vec{n}} \wedge \vec{A}(\vec{n})=\vec{n},
$$

the DB between the components of $\vec{n}$ reads:

$$
\left\{n_{i}, n_{j}\right\}_{D B}=\frac{n_{k}}{s} .
$$

In the last expression ijk are the integers 123 and its cyclic permutations.

If we now identify $s \hat{n}_{i} \rightarrow \hat{S}_{i}$, we obtain:

$$
\left[\hat{S}_{i}, \hat{S}_{j}\right]=\imath \hbar \hat{S}_{k}
$$

which corresponds to the commutation relations of the components of the spin operator. Then, we have proposed a classical constrained theory which after the Dirac procedure reproduce the commutation relations for the spin.

Now, we are able to quantize the system using Feyman path integral. We shall use the method developed for constrained systems in [9]. In this method the probability amplitude of the system which was at $\overrightarrow{n_{0}}$ at $t=0$ and will be at $\overrightarrow{n_{1}}$ at time $T$ can be written as:

$$
<\overrightarrow{n_{0}} \mid \overrightarrow{n_{1}}>=\int d \vec{n} d \vec{P}\left(\prod_{i} \delta\left(\Gamma_{i}\right)\right)\left(\operatorname{det} \Delta^{-1}\right)^{1 / 2} \exp \left(\frac{\imath}{\hbar} \int_{0}^{T} d t\left[\vec{P} \overrightarrow{\vec{n}}-H_{T}\right]\right)
$$

Using the fact that $\operatorname{det}(\Delta)$ is a constant independent of the fields (see (10)) and integrating over $\vec{P}$, we obtain

$$
<\overrightarrow{n_{0}} \mid \overrightarrow{n_{1}}>=\int d \vec{n} \delta\left(|\vec{n}|^{2}-1\right) \exp \left(\frac{\imath}{\hbar} \int_{0}^{T} d t[s \vec{A}(\vec{n}) \dot{\vec{n}}-H(\vec{n})]\right) .
$$

To obtain the partition function, we must carry out the integration over all periodic paths, make the change $i t=\tau$ and identify $i T / \hbar$ with the inverse of the temperature $\beta$ [10. Thus we have

$$
Z=\int_{\text {periodic }} d \vec{n} \delta\left(|\vec{n}|^{2}-1\right) \exp \left(-\frac{1}{\hbar} \int_{0}^{\beta \hbar} d \tau\left[-s \vec{A}(\vec{n}) \frac{d \vec{n}}{d \tau}+H(\vec{n})\right]\right) .
$$


Equations (16) and (17), with $\vec{A}$ given by (12), are exactly the expressions for the path integral for the spin system obtained via coherent states [6].

Before going to the next section it is useful to make a few remarks about the reason for choosing the set of constrains (2)-(5). Our principal aim was to find a classical system containing the nature of the spin. To get a chance to find that, the classical system must contain constraints, and these constraints must be second class. The set of constraints (2)-(5) are arbitrary functions of the coordinates and momenta and our previous results showed that after imposing condition (12) the quantized theory corresponds to the spin system. On the other hand, the classical constrained system we have introduced describe a system of massless particles living on unitary spheres. Each particle feels a magnetic field represented by the vector potential $\vec{A}$. This vector potential corresponds to the one of a monopole placed at the center of the sphere as is stressed by condition (12). This mechanical analogy was previously suggested from the path integral expression obtained via coherent states [6] and has indeed inspired the election of our set of constraints. It is important to point out that once the constrained Hamiltonian system was defined we were able to show that the path integral representation is valid for any parameter $s$. This is a point not allways clear in the literature.

\section{An example}

Our starting point is eq.(17), with the Hamiltonian

$$
H(\vec{n})=-s \vec{n} \cdot \vec{B}
$$

The first term in the action of eq.(17) can be written, with the aid of eq.(12) and Stoke's theorem, as

$$
\int_{0}^{\beta} d t \vec{A}(\vec{n}) \cdot d_{t} \vec{n}=\iint \vec{n}(t, \tau) \cdot d \vec{a}=\int_{0}^{\beta} d t \int_{0}^{1} d \tau \vec{n} \cdot \partial_{t} \vec{n} \times \vec{n} .
$$

$n(t, \tau)$ is a parametrization of the surface on $S^{2}$ enclosed by the trajectory $\vec{n}(t)$, that we chose so that $\vec{n}(t, 0)=\vec{n}(t)$ and $\vec{n}(t, 1)=\breve{k}$. We then see that this term has a geometrical interpretation, which corresponds to the

oriented area on the sphere $S^{2}$ enclosed by $\vec{n}(t)$. Terms of this kind are known as Wess-Zumino terms. 
Using the explicit parametrization

$$
\begin{aligned}
& \vec{n}(t, \tau)= \\
& \quad(\sin [(1-\tau) \theta(t)] \cos \phi(t), \sin [(1-\tau) \theta(t)] \sin \phi(t), \cos [(1-\tau) \theta(t)])(20
\end{aligned}
$$

where $t \in[0, \beta], \tau \in[0,1]$, the surface element is then given by

$$
\vec{n} . \partial_{t} \vec{n} \times \vec{n}=\dot{\phi(t)} \theta(t) \sin [(1-\tau) \theta(t)] .
$$

The use of spherical coordinates for $\vec{n}$ allows for a straightforward implementation of the constraint $\vec{n}^{2}=1$ and the functional measure is

$$
\mathcal{D} \vec{n}(t) \delta\left(\vec{n}^{2}-1\right)=\mathcal{D} \varphi(t) \mathcal{D}(\cos \theta(t)) .
$$

Making use of the coordinates (20), the partition function (17) can be written as

$$
\mathcal{Z}=\mathcal{D} \varphi(t) \mathcal{D} u(t) \exp \left(\imath s \int_{0}^{\beta} d t \dot{\varphi}(t)(1-u(t))-s B \int_{0}^{\beta} u(t) d t\right)
$$

where $u(t)=\cos (\theta(t))$, the integral over $\tau$ has been carried out in the WZ term and the functional integration is restricted to continuous closed trajectories. The corresponding boundary conditions are

$$
\varphi(\beta)=\varphi(0)+2 k \pi, \quad \theta(\beta)=\theta(0)+2 k^{\prime} \pi
$$

and

$$
\varphi(\beta)=\varphi(0)+(2 \tilde{k}+1) \pi, \quad \theta(\beta)=-\theta(0)+2 \tilde{k}^{\prime} \pi .
$$

We will first integrate out $\varphi(t)$. It is convenient to integrate by parts the first term in the exponent of (23). Then it reads

$$
\imath s(\varphi(\beta)-\varphi(0))(1-u(0))+\imath s \int_{0}^{\beta} \varphi(t) \dot{u}(t) d t .
$$

The change of $\varphi$ along the trajectory, $(\Delta \varphi=\varphi(\beta)-\varphi(0))$, is a constant $2 k \pi$ and $(2 \tilde{k}+1) \pi$ for trajectories satisfying (24) and (25) respectively.

The functional integral over $\varphi$ is now straightforward and the result is 


$$
\int \mathcal{D} \varphi(t) \exp \left(i s \int_{0}^{\beta} d t \dot{\varphi}(t)(1-u(t))\right)=\sum_{\Delta \varphi} \delta[\dot{u}(t)] \exp \left(\imath s \Delta \varphi\left(1-u_{0}\right)\right) \text {. }
$$

The $\delta$-functional restricts the integral over $u(t)$ to constant trajectories. For this reason any trajectory satisfying the boundary conditions (25) is wiped out from the partition function. The functional integral over the remaining trajectories can be written as a sum of ordinary integrals, one for each posible value of $\Delta \varphi$.

$$
\begin{gathered}
\mathcal{Z}=\sum_{k} \int_{-1}^{1} d u_{0} \exp \left(i s 2 k \pi\left(1-u_{0}\right)-s B \beta u_{0}\right)= \\
\int_{-1}^{1} d u_{0} \sum_{-\infty}^{\infty} \delta\left(s\left(1-u_{0}\right)-n\right) \exp \left(-s B \beta_{0}\right) .
\end{gathered}
$$

The second equality follows from the Fourier representation of the $\delta$-function.

Integrating now over $u_{0}$ gives

$$
\mathcal{Z}=\sum_{0}^{2 s} \exp (-\beta B(s-r))
$$

which can be rewritten as

$$
\mathcal{Z}=\sum_{m=-s}^{s} \exp (-\beta B m)
$$

This expression for the partition function coincides with the well-known result obtained in elementary quantum statistical mechanics.

It should be stressed that our result was obtained without the use of any approximation within the path-integral approach. One sould recall at this point that one of the mathematical subtleties involved in the formal construction of path-integrals is the restriction to continuous trajectories. Our computation, which explicitly uses this restriction, provides a check that this procedure leads to the correct result.

It is important to point out that at the classical level there is no reason to choose the parameter $s$ to be integer or half-integer. In this section we have found that this condition emerges naturally when the path integral for a 1-site problem is solved. 


\section{Summary}

In this paper we have presented a constrainted Hamiltonian theory for spin systems. Using the Dirac theory we have shown that our classical system has DB which can be identified with the commutation relation for the components of the spin. Following the recipe for quantizing constrained theories we have obtained a path integral representation for the spin system, which coincides with the expression obtained using coherent states [6].

Our approach shows in a clear way that the path integral for the spin systems is free of any approximation.

Finally, we have also recovered the partition function for a single spin in a magnetic field from our path integral expression.

Natural extensions of the present work would be to study the case of $O \operatorname{sp}(2,2)$ (related to the t-J model) and $S U(3)$ groups. In fact, we are working actually on this problem, but so far we have found that a generalization of this work is not straightforward and deserves a separate treatment.

\section{Acknowledgments:}

The authors acknowledge Fundación Antorchas for finantial support. A.G. and A.D. would like to thank C. Abecasis, A.Foussats and O.Zandron for valuable discussions. D.C. thanks F. Schaposnik for a careful reading of the manuscript. 


\section{References}

[1] Hirsch J.E. (1985) Phys. Rev.Lett 541317

[2] Hubbard J. (1963) Proc. R. Soc. London Ser. A276,238

[3] Wiegmann P.B. (1988) Phys. Rev.Lett 60821

[4] Auerbach A. (1994) Interacting electrons an quantum magnetism, Sringer Verlag

[5] Greco A. and Zeyher R. (1996) Europhysics Lett. 35115.

[6] Fradkin E. and Stone M. 1988 Phys. Rev. B 17 7215. Fradkin E. 1991 Field Theories of Condensed Matter Systems, Addison-wesley publishing company.

[7] Shankar R. (1990) Nuclear Phys. B 330433.

[8] Dirac P 1964 Lectures on Quantum Mechanics (New York: Academic).

[9] Senjanovic P 1976 Ann. Phys. NY 100227.

[10] Bernard C 1974 Phys.Rev D 93312. 